\title{
Le droit au rater dans le contexte artistique athénien
}

Angeliki Poulou, Estelle Baudou et Quentin Rioual

\section{OpenEdition}

1 Journals

Édition électronique

URL : https://journals.openedition.org/agon/8593

DOI : 10.4000 /agon.8593

ISSN : 1961-8581

Éditeur

Association Agôn

Référence électronique

Angeliki Poulou, Estelle Baudou et Quentin Rioual, « Le droit au rater dans le contexte artistique athénien », Agôn [En ligne], 9 | 2021, mis en ligne le 12 janvier 2022, consulté le 18 janvier 2022. URL : http://journals.openedition.org/agon/8593; DOI : https://doi.org/10.4000/agon.8593

Ce document a été généré automatiquement le 18 janvier 2022.

Association Agôn et les auteurs des articles 


\title{
Le droit au rater dans le contexte artistique athénien
}

\author{
Angeliki Poulou, Estelle Baudou et Quentin Rioual
}

\section{NOTE DE L'ÉDITEUR}

Entretien avec Angeliki Poulou réalisé par visioconférence le 05 juin 2020, mené par Estelle Baudou et Quentin Rioual.

\section{Les ratés de la crise sanitaire}

\begin{abstract}
AGôn: Depuis trois mois, le monde est confronté à une pandémie ayant conduit plusieurs pays, notamment européens, à confiner la majeure partie de la population. En conséquence, toutes les activités dites non essentielles sont interdites, à commencer par le théâtre. De votre point de vue, qu'est-ce que la crise sanitaire a fait rater au théâtre et aux artistes grecs de théâtre?

ANGELIKI POULOU: Pour les artistes, les travailleuses et travailleurs du théatre grec que's, la crise sanitaire a associé pour la deuxième fois en dix ans, et de façon massive, l'instabilité de leur art, la précarité financière et la perte d'un sentiment de sécurité. La crise financière mondiale avait, en Grèce, constitué le premier terreau d'expérimentation d'une telle fragilité généralisée. À cela, s'est jointe cette fois une crise peut-être plus existentielle : de façon radicale, l'espace même où l'artiste peut performer sa grandeur était rendu inaccessible, et ce en dépit de tous les efforts possiblement concédés. L'estime de soi, parfois la confirmation de soi, et l'inclination intérieure quotidienne se sont étiolées. Certain'e-s en ont toutefois profité pour se préoccuper des raisons qui les poussaient à faire de l'art et mettre, ou remettre, sur le métier leur réflexion sur les conditions de production de leur travail. Autant il me semble que la majorité a accepté d'avoir annulé, reporté et donc en un sens raté ce qui était prévu pour elles et pour eux, autant il me semble que nous y avons aussi perdu un certain esprit collectif qui existait avant la crise.
\end{abstract}


Il est remarquable que la plupart des organisations théâtrales en Grèce annoncent désormais un "répertoire numérique » pour rester actives pendant la pandémie et le confinement. Or, le slogan ne devrait pas être « répondons numériquement à la crise sanitaire » mais « répondons politiquement ». Par là, je veux dire que le digital et la digitalisation ne devraient pas être $a$ priori la solution. Il me paraît nécessaire, plutôt, de chercher des micro-alternatives, des actions "artisanales » basées et adressées aux communautés locales, aux micro-espaces extérieurs. La solution pour ne pas s'éloigner du spectateur, n'est pas d'envoyer le spectateur - déjà enfermé à cause du télétravail - à voir et entendre les œuvres sur son écran, mais de l'inviter à « regarder » vers l'extérieur, vers l'horizon et l'espace physique, de le soutenir pour réinventer avec lui l'espace physique et matériel.

AGôn : Vis-à-vis de la première crise - financière, telle qu'usuellement résumée -, dont la puissance en Grèce a été toute particulière, cette deuxième crise - d'abord sanitaire vous paraît-elle constituer un infléchissement particulier?

A. P. : Les artistes ont en fait réalisé que rien n'avait changé depuis la première crise. Ils se sont rendu compte que, pendant dix ans, ils n'avaient presque rien revendiqué ensemble, sinon de l'argent. Aucun changement en profondeur. Au cœur de la crise financière, chacun a d'abord cherché à conserver ou retrouver une visibilité, c'est-àdire à faire en sorte que sa carrière individuelle reprenne, en investissant beaucoup sur les connaissances et les réseaux. Depuis 2010, dans les milieux artistiques athéniens dont je suis familière, la plupart des spectacles ont ainsi été produits avec un budget extérieur minimal. Les artistes se sont auto-financé.e.s, ont fait financer leur travail par leurs proches, et la majorité a accepté tout à la fois de ne pas être rémunérée pour le travail de répétitions ou, quand ce n'était pas le cas, d'être rémunérée hors de tout cadre légal. En somme, la crise sanitaire a révélé aux artistes de théâtre grecs ce qu'ils ont raté, entre elles et eux, dans les années qui ont suivi la crise financière. Elle manifeste, à mon sens et au sens des nombreux témoignages que j'ai pu entendre, une défaite de la communauté des artistes de théâtre grec'que·s qui n'a pas su ou pu se reconstruire solidairement.

Paradoxalement, en plein cœur de la crise sanitaire, nous avons assisté à un début de retravail de l'identité des artistes comme travailleurs, et non plus comme sortes de « chamans » devant bénéficier d'un statut spécial en-dehors de la collectivité. C'est pourtant ce statut qui avait été revendiqué pendant la crise économique. La stratégie, cette fois, a consisté à demander au Ministère de la culture grec d'être identifié comme des artistes-travailleurs afin de pouvoir imposer l'ensemble du cadre légal relatif aux rémunérations. Le Ministère de la culture a renvoyé ces artistes voulant être identifiés comme des travailleurs vers le Ministère du travail, considérant que son rôle n'était pas de définir qui est artiste ou qui ne l'est pas.

AGôn : Le Ministère de la culture grec rate-t-il quelque chose avec cette décision?

A. P.: Comme avant la crise, le Ministère de la culture a manqué là une occasion de réduire la grande distance qui le sépare, d'une part, de la société, d'autre part des artistes eux- et elles-mêmes. Cette situation est notamment le résultat d'une absence de politique culturelle et, par conséquent, de stratégie culturelle définie à l'échelle ministérielle. Les artistes et les lieux indépendants sont en péril, et leur Ministère n'est pas en mesure ni de les aider à porter les débats ni à instruire les enjeux à venir, notamment ceux posés à leur art vivant par le confinement. Par comparaison, le 
Ministère de la culture grec assure donc très peu des rôles qu'assure le Ministère de la culture français.

En l'occurrence, pendant le confinement, les artistes grecs ont beaucoup présenté le modèle français comme un exemple à suivre. Précisément, les mesures relatives à la prolongation de l'intermittence ont été perçues comme une marque de respect des artistes dont les Grec.que.s manquent cruellement. Or, nous savons aussi que ce respect est dû à l'important travail effectué par des associations professionnelles dont la Grèce manque aussi. À titre d'exemple, il n'existe pas chez nous de statut comparable au statut d'intermittent. Ce qui s'y rapproche le plus est une allocation inférieure au SMIC attribuée pour certains mois, sur la base de plusieurs paramètres.

Pour bien comprendre la relation de tension entre le Ministère et les artistes, il me semble important de préciser que l'État n'est généralement pas, en Grèce, considéré comme pouvant être de confiance. Cela tient à des raisons historiques multiples, et notamment au fait que l'État grec n'a pas été constitué par des citoyen'ne'srésident·es grec'que's mais bien plutôt par des forces extérieures. De son côté, l'État paraît considérer que les artistes manquent de reconnaissance et de fidélité à son égard. Le dialogue en est nécessairement compromis.

\section{S'hybrider, s'augmenter, se perdre}

AGôn: Aujourd'hui, votre réflexion sur la situation des artistes de théâtre en Grèce, et plus spécifiquement à Athènes, se fonde sur plusieurs expertises. Celles-ci proviennent du fait que vous cumulez différentes activités, cumul qui est caractéristique d'une obligation à s'hybrider pour travailler dans le champ artistique et pour pouvoir en vivre. Pourriez-vous présenter ces activités?

A.P.: Pour présenter le plus petit cadre de cumul d'activités, je me contente d'identifier celles qui me permettent de gagner ma vie. Je suis donc tout à la fois academic fellow au département des arts numériques et du cinéma de l'Université d'Athènes (poste à peu près équivalent à un ATER en France), curatrice académique à la fondation Michael Cacoyannis depuis 2013 et dramaturge indépendante pour plusieurs compagnies de spectacle vivant. Lorsque je postule à un emploi ou lorsque je dépose une demande de financement, c'est chaque fois un casse-tête: dois-je utiliser le titre de " chercheuse », d' « artiste », de « dramaturge » ou encore un autre rendant davantage compte de tout cela ensemble?

AGôn : Diriez-vous que ces différents métiers vécus en même temps vous font rater quelque chose?

A. P. : Assurément, ils ne me laissent aucun temps à moi-même. Je suis constamment dans l'urgence et je sais que je me résous parfois à devoir être superficielle. Par exemple, alors que je n'avais absolument aucun temps pour moi, j'ai accepté, un jour, d'écrire un texte pour l'exposition « Metaphoria II » réalisée à Athènes, par Lab'Bel où j'avais travaillé comme médiatrice culturelle. Une nuit, je me suis réveillée pour corriger tout le texte que j'avais envoyé quelques heures plus tôt. J'ai demandé à avoir du temps en plus, à dépasser l'urgence imposée, en expliquant qu'on a toujours besoin de temps pour élaborer nos idées.

Je rate la magie des choses. Et, pour une artiste, ce n'est pas peu de dire cela. Je travaille en effectuant des tâches. Je coche des cases. J'ai perdu l'énergie de participer 
aux temps de réflexion en équipe, de développer des discussions longues. Je faisais cela à Paris lors de mes années de master et de doctorat. J'avais du temps pour interagir avec des gens, et surtout du temps pour dériver. Ce n'est plus le cas. Et certain'e's de mes ami-e's ont le même problème. En somme, nous vivons notre vie en tant que projet où il s'agit de tout contrôler, tout savoir, sans jamais aucune légèreté.

Comme l'affirme Olivier Gausselain dans son article emblématique «Slow Science La désexcellence ", il en résulte une baisse marquée de la spéculation intellectuelle et de la créativité. Le formatage des projets commence d'ailleurs, d'après lui, dès le niveau doctoral. Pour espérer un financement, les jeunes chercheurs

se trouvent obligés de proposer des recherches balisées, qui sortent aussi peu que possible des sentiers battus. Annonçant pratiquement leurs résultats à l'avance, ils tentent ainsi de garantir le retour sur investissement. S'il fallait évaluer les projets de la génération précédente sur de telles bases, la plupart seraient tout simplement refusés. Quelle ironie, quand on pense que ses représentants occupent aujourd'hui les postes d'évaluateurs. Dans cette perspective, refuser l'excellence c'est refuser une politique scientifique qui nous condamne à l'égoïsme, au calcul et à la médiocrité. Qui nous oblige à être acteurs de notre propre destruction. Car ne nous y trompons pas : la bureaucratisation et la dérive managériale du monde académique ne sont pas le fait d'acteurs externes ${ }^{1}$.

En art, la transition depuis le paradigme de l'œuvre vers celui du projet ne me paraitt pas insignifiante. Aujourd'hui, le mot « projet » a remplacé le concept d'œuvre. Voilà le langage artistique de notre ère : State of the art, work plan, work unit, work packages, deliverables, measures, impact. Tout est bien calculé, dans les délais et tout en respectant le budget. Les artistes et les chercheure's sont appelée's à produire des projets, pas des œuvres. La culture actuelle, envahie par le langage du marketing, a traduit l'œuvre d'art en critères mesurables. Les artistes sont invitée's à décrire avec précision leur « œuvre/projet » avant de créer, à un niveau tel qu'ils doivent prédire et contrôler ce qu'ils vont produire. Ils doivent prévoir les ratés, les incidents, les retards possibles, et proposer des mesures pour pouvoir y répondre. Ils doivent démontrer, en somme, que leur proposition est réalisable et qu'elle ne laisse pas de place à l'erreur.

La mobilisation du projet sous ses différentes facettes dans la plupart des univers du social caractériserait notre société (Boutinet, 1993) et serait au fondement du capitalisme actuel, expression du monde des réseaux et des structures labiles (Boltanski et Chiapello, 1999). C'est un processus qui modifie à la fois le concept (la signification et l'ontologie) de l'œuvre d'art (si nous en parlons encore), le rôle de l'artiste et les conditions de pratiquer son art. Cette tendance va, d'après moi, de pair avec la tendance générale de l'ère : nous n'avons pas d'idéal d'émancipation politique collective comme ceux du XXe siècle. Les Grands Récits sont considérés comme finis, laissant la place à l'individu, au fragment, au non-sens. Dans cette perspective, tout commence, rien ne se termine. Tout est déjà fait. Tout est une déclaration d'intention. Nous ne nous soucions plus de raconter une histoire, de donner un sens, quand bien même celui-ci serait éphémère. Les projets sont donc individuels d'une part, limités dans le temps d'autre part.

AGôN: À l'inverse, pourrait-on envisager que cumuler ces différentes activités vous permettent de réussir certaines choses?

A. P. : Je sens que jongler entre ces différents métiers et avoir développé une habileté dans plusieurs champs me conduit à pouvoir argumenter de tout, du moins à avoir 
l'impression qu'il m'est possible de le faire. J'élabore, je construis, je dissimule de sorte à faire se correspondre un projet et un appel qui jouent aux dupes ou, pire, participent d'un cynisme caractéristique de la postmodernité. Car, si l'on remporte cet appel, le projet de spectacle sera bricolé de sorte à correspondre à un "mensonge ", au mieux à un compromis qui me parait se faire au détriment d'une idée de l'art, des artistes eux-mêmes et nécessairement des spectatrices et des spectateurs.

Parfois, en réfléchissant à cette panoplie de fonctions que j'occupe, il m'arrive de me dire, pour me faire un peu de bien : "ah bah, en fait, Angeliki, c'est ça, tu es une artiste de la Renaissance! Tu peux tout jouer, tout faire.» Mais ce n'est pas vrai. J'agis comme une bricoleuse. La polyvalence actuelle n'a rien à voir avec l'esprit total, universel et humaniste qui constitue notre représentation de l'artiste de la Renaissance. Parce que, moi, je pense rater l'essentiel - comme d'autres, sans doute. Je n'ai, nous n'avons pas de temps pour créer de nouveaux regards pour le cosmos, pour la vie, pour l'être humain. Or, en tant qu'artiste, je veux qu'il s'agisse là de ma contribution principale!

AGôn: Diriez-vous que ces nouveaux regards sont impossibles dans le temps car un.e tel.le artiste est nulle part : ni dans un temps, ni dans un lieu?

A. P.: Un.e tel.le artiste me semble ne pouvoir apporter que des réponses éphémères à de mêmes questions - parfois elles-mêmes éphémères. Par exemple, à la question du post-Covid posée aux artistes, la majorité des témoignages a traduit l'impossibilité de se projeter. Les revendications n'avaient pas bougé et visaient principalement des enjeux financiers, ce qui devient typique lorsque l'on passe le plus clair de son temps à se vendre pour acheter du temps que l'on perd dans l'acte même de l'autopromotion et de la vente d'un projet consensuel.

Que dire en outre d'une projection qui se fait majoritairement dans une pensée par le « post-» : post-Covid, post-crise sanitaire, post-démocratie, post-modernité... Malheureusement, il me semble que ces termes traduisent une gêne, sinon une impossibilité, à définir les choses et à suggérer des horizons. Nous n'avons en somme que des petites histoires définies à partir de choses qui ne sont plus : idées, états du monde... On n'a pas encore trouvé comment définir notre ère. Or, d'après moi, une avant-garde ne se mesure pas aux formats qu'elle crée mais d'abord aux nouveaux regards que ces formats induisent. Il me semble très compliqué d'y parvenir alors que l'artiste est rendu comme manager de lui-même et enraciné nulle part. À titre personnel, il en résulte un manque de confiance en moi et le sentiment d'être en l'air. Parfois, je me retrouve fatiguée avant même la réalisation d'un projet. Je veux à la fois percuter les notions d'innovation et de nouveauté mais suis contrainte de vendre mon travail avant même d'avoir pu le faire.

D'après moi, cela participe du « capitalisme émotionnel », dont parle Eva Illouz dans son ouvrage Les sentiments du capitalisme $e^{2}$. Le capitalisme émotionnel pousse l'individu à la réalisation la plus intime de soi d'une manière plus qu'ambiguë : il s'approprie les affects au point de transformer les émotions en marchandises, ce qui culmine aujourd'hui dans les nouvelles formes de sociabilité nées de l'Internet. Le capitalisme émotionnel est une culture dans laquelle les discours émotionnel et économique ainsi que les pratiques correspondantes sont mutuellement transformés. Le capitalisme émotionnel a réorganisé les cultures émotionnelles en transformant le soi économique en soi émotionnel, et en instrumentalisant les émotions. Ainsi la vie 
émotionnelle suit-elle la logique des relations économiques et fait-elle de l'émotion un aspect important de la vie économique. Le marché donne corps aux relations interpersonnelles et émotionnelles, tandis que les relations interpersonnelles se trouvent désormais au cœur des relations économiques. En conséquence, le langage de la psychologie a façonné avec succès le monde des affaires lui-même... et vice versa.

\section{Le droit au rater dans le contexte artistique athénien}

AGôn : Comment sont structurées en Grèce les revendications à de nouvelles conditions de production artistique et à un droit au rater?

A. P. : Des associations portent ce message " percussif » et demandent à réinventer le système de production artistique, à l'instar de "Support Art Workers » ou de «Cultural Workers Alliance Greece ». Au sein des institutions, il y a quelques espaces établis sur un modèle de production revisité comme le Viz Laboratory for Visual Culture ou le Projet Occupy \#2 et «Carte blanche » de l'Institut culturel français ${ }^{3}$. Le discours le plus radical est porté par des lieux indépendants. Les artistes qui s'y produisent font plutôt des spectacles courts et à petit budget. Leur position est celle de créatrices et de créateurs désirant construire des lieux d'indépendance et de recherche dont les processus peuvent ne pas aboutir à des résultats.

Ces lieux sont peu nombreux ${ }^{4}$ mais ils ont un succès notable. La scène underground d'Athènes existe grâce à ces artistes qui, pour certain'e's, sont des Grec'que's revenu·e's d'un séjour en Allemagne, en France ou aux États-Unis. L'espace que ces lieux occupe est à mon sens essentiel. Ils permettent que tout le temps ne soit pas perdu à contester toujours, mais bien à proposer.

AGôn : Quelle relation entretiennent ces lieux avec les fondations privées dont le rôle semble se substituer pour partie à celui du Ministère de la culture?

A. P.: Les fondations privées ont repéré ces lieux et, dans la mesure où elles cherchent toujours à trouver de nouvelles voies, elles les ont bien approchés pour leur proposer de collaborer. La réponse n'est pas toujours positive de la part des lieux indépendants, qui craignent que ce type de collaboration les normalise. Du point de vue des projets, certaines fondations n'imposent pas de critères, comme les fondations Onassis et Niarchos. Pour autant, elles jouent le rôle d'un régulateur de la culture et du goût, avec une étonnante liberté de financement.

Dans ce paysage, un débat entre artistes est né qui n'a pas trouvé sa réponse définitive pour le moment: que faire si les fondations te proposent une collaboration? Être soutenu par des fondations qui sont possédées par des compagnies maritimes historiques est loin d'être anodin au niveau idéologique. Certain'e's artistes refusent d'ailleurs et appellent à refuser. D'autres avancent, aussi légitimement, me semble-t-il, qu'il s'agit de leur unique moyen de pouvoir faire de l'art, donc de pouvoir vivre et de pouvoir exister en tant qu'artistes. Le problème principal n'est pas l'activité des fondations mais l'absence du Ministère de la culture. Les fondations privées font leur travail, et le font très bien, avec des résultats réjouissants: elles contribuent à ce que se tiennent des débats théoriques, elles introduisent en Grèce des artistes de l'étranger. Pendant la crise financière, ce sont 
elles qui ont soutenu largement les artistes et qui ont financé des productions coûteuses.

Donc il s'agirait de porter notre attention et nos revendications vers le Ministère de la culture qui a priori est chargé du champ culturel en tant que bien commun. D'après moi, c'est une faute du Ministère de la culture d'avoir laissé le théâtre sans stratégie et sans argent public.

En l'occurrence, il m'est arrivé de travailler plusieurs fois avec la fondation Onassis. J'ai moi-même été régulièrement critiquée par mes proches car i-elles estimaient que j'aidais cette fondation à se créer une belle image, à l'intersection de champs économico-politique et artistique. Il n'en reste pas moins qu'en l'état ces fondations occupent un vide et promeuvent des sujets engagés, aussi marketing que soit ce positionnement : la théorie queer, les commons, les nouveaux médias, les réfugié'e·s... Le Ministère de la culture, dont l'ambition semble être de traiter des grands monuments et des sujets passéistes, en est loin. Cependant, je dois mentionner que le Ministère de la Culture offre depuis quelques années un nombre limité de subventions à des compagnies de théâtre et de danse, et qu'en avril 2020 et en janvier 2021 il a lancé une série d'appels concernant la culture numérique. Peut-être s'agit-il là d'un timide réveil ?

AGôn : Dans quelle mesure ces fondations peuvent-elles autoriser un droit au rater?

A.P.: Sur ce point, il ne me semble pas y avoir de grande différence entre les fondations et les lieux indépendants. Je n'ai en effet jamais connu de censure de la part des fondations. Medea Electronique, compagnie dont je fais partie, a régulièrement été financée par l'Institut français, par la fondation Onassis et par la fondation Cacoyannis. Ces fondations ont des studios, des salles de répétition, des salles de théâtre, et elles invitent les artistes à occuper ces espaces. Il n'en reste pas moins que c'est un sujet de vigilance et de discussion permanent, dans un contexte où de nombreux'ses artistes cherchent à être financée's de manière constante par les fondations ou par le Ministère de la culture, et où l'on aimerait envisager qu'une frange plus importante d'artistes puisse ne pas chercher cela mais se construire au travers de lieux radicalement indépendants.

AGôn : Pourriez-vous décrire le cas d'un·e artiste qui serait exemplaire du point de vue de l'affirmation à un droit au rater?

Célèbre en Grèce, l'artiste Lena Kitsopoulou écrit pour le théâtre, la poésie et a comme singularité d'associer souvent la musique rebetiko à ses travaux. La fondation Onassis, dont on pourrait dire que Lena Kitsopoulou est une "enfant gâtée ", l'a notamment aidée à diffuser son activité à l'étranger. Cette artiste bien installée s'est fait remarquer pendant le confinement, à l'occasion d'une attribution de la fondation Onassis proposant aux artistes qu'ielles produisent quelque chose à partir des moyens numériques dont ils disposaient. La seule contrainte consistait à créer dans un délai de $120 \mathrm{~h}$. Dans ce cadre, Lena Kitsopoulou a réalisé quelque chose qui a fait beaucoup débat : une vidéo très artisanale et, on pourrait dire, superficielle, très peu travaillée en apparence ${ }^{5}$. Sans atours et sans filtre, elle y provoque le spectateur dans une vidéo dont le fil rouge consiste à exposer comment elle tue les animaux et les cuisine... Désigné comme victime de la propagande, le spectateur me semble invité à un comportement dionysiaque somme toute assez consensuel : «Si vous voulez vivre, vous devez fumer ! ». Partie vivre le confinement à la montagne, elle décrit avoir pris 
conscience de son animalité, elle, l'artiste abondamment subventionnée, se disant plus humaine en tuant là-bas des animaux que, nous, ici, en nous « tuant » les uns les autres.

Par la médiocrité manifeste de la réalisation et par ce ton politiquement incorrect finalement consensuel, j'ai le sentiment que cette artiste a fait son coming out dans son droit au rater. Et de fait, la plupart des gens sont très critiques à son égard depuis cette vidéo. Pour avoir pris ce risque, je trouve pour ma part que c'est un beau raté d'une artiste confortablement située dans le champ théâtral grec. Pour utiliser les mots du Comité Invisible, c'est comme si Lena Kitsopoulou dénonçait là le système de production moderne, cette machine géante de vigilance psychologique et physique, de consommation d'énergie humaine, cette machine de tri qui n'accorde le droit de survivre qu'à des subjectivités conformes.

AGôn : Cette crise sanitaire ayant fait rater tous les spectacles en présentiel, cette réponse pourrait être vue comme une forme de "Regardez, on n'a plus rien et vous voulez qu'on fasse quelque chose? Eh bien, je vous fais du médiocre en ligne! »

A. P.: Tout à fait. Et c'est ce qui a pu apparaître comme une forme de condescendance à l'égard d'artistes qui ne peuvent se permettre cette désinvolture. Or, précisément, ce peut être une forme de porte-parole d'une exaspération à la réponse aux appels, même en période critique. À l'opposé de ce spectre, l'artiste Alexandros Mistriotis serait l'archétype de celui qui se donne à lui-même le droit de rater et en fait une éthique artistique entière. L'un de ses plus précieux combats repose sur la critique de l'idéalisme entourant l'imaginaire antique de la Grèce contemporaine. The Reception, promenade poétique et historique dans le centre d'Athènes, en est un parfait exemple dans la mesure où l'enjeu était de remettre en cause la construction historique de l'identité grecque à travers l'idéalisme allemand. Récemment, il a achevé un long texte poétique sur Athènes, texte auquel il travaillait depuis plusieurs années. Pour, de sa propre voix, lire ce texte aux auditrice-teur.s, il a d'ailleurs lancé une radio sur le web6.

AGôn : Dans quelle mesure cet idéalisme pèse encore dans le champ artistique grec et fait rater leur intention initiale ou leur cible à certaines manifestations ?

A.P.: On ne peut nier, je crois, le fait que perdure chez de jeunes compagnies grecques un sentiment de colonialisme culturel auquel participent les grands événements d'institutions binationales ou étrangères. De ce point de vue, la Documenta 14 fut caractéristique. Son titre: "Learning from Athens ». Il s'agissait d'apprendre d'Athènes, donc, de tirer les enseignements de cette ville. Un grand programme poétique et politique, en somme. Le modus operandi de la manifestation consistait à inviter des artistes à s'inspirer d'Athènes en l'explorant, dans l'objectif de créer des œuvres in situ dans la capitale. Les œuvres produites étaient intéressantes mais n'avaient presque rien à voir avec Athènes ou ne s'intéressaient qu'à ses ruines. Or très peu d'artistes grec'que's ont été mobilisée's ou invitée's, ce qui n'a sans doute pas contribué à contrecarrer un regard essentiellement exotique sur la capitale. Un contre-exemple très positif est celui offert par le travail « The Tempest Society » de Bouchra Khalili qui a fait un documentaire entier sur les immigrants en Grèce, après deux années de travail sur le terrain. Il me semble que Bouchra Khalili, contrairement à celles et ceux qui s'intéressent aux ruines d'Athènes, aspirait à explorer la «mise en ruines » actuelle et non les ruines. Les artistes programmée's avaient évidemment des intentions, des obsessions, leur style, mais ils ne semblaient 
pas vouloir explorer Athènes. Plutôt, ils semblaient vouloir être accueillis par Documenta. Et je suis la première à les comprendre au vu des conditions de production, du fonctionnement par appel... On cherche à faire passer nos projets originaux dans des appels qui ne le sont pas. Chacun se force la main, au risque de la tordre ou de manquer la poignée.

Pour le philosophe français Jacques Rancière, la radicalité artistique et l'utopie esthétique et avant-gardiste sont remplacées par le nouveau système du branding, par un art modeste, non seulement par rapport à sa capacité de transformer le monde, mais par rapport à la confirmation de l'unicité de ses objets. Ces situations insignifiantes alors créées ne s'écartent guère de celles de la vie ordinaire. Elles sont majoritairement présentées de manière ironique et ludique plutôt que critique et plaintive. Elles ne visent plus à créer ou recréer des liens entre les individus, ni à donner naissance à de nouveaux modes de confrontation et de participation.

En conséquence, il m'apparaît clair que, ici, trois choses (Athènes, l'artiste, la Documenta) se sont ratées. Et j'aurais tendance à dire que ce type de grand événement, très financé, peu implanté, laisse l'impression malheureuse que, en dépit de tous les efforts, humains et financiers, rien ne se rencontre réellement.

On a besoin du retour des Grands Recits sur la scène artistique, de l'invention de nouvelles mythologies et de nouvelles dramaturgies. Que la scène soit pleine d'œuvres et pas de projets! Que le théâtre se lance vers le Chaos et le Mythe, occupé par des signes de rupture et de fuite, offrant de nouvelles spatialités, temporalités, méthodologies, atmosphères! Que l'art soit à nouveau "utopique", qu'il soit ce nonlieu où l'ordre mondial donné est mis en désordre expérimental!

À la fin et sans doute - comme Olivier Neveux l'affirme dans son ouvrage Contre le théâtre politique ${ }^{7}-$, il serait triste de demander du grand art et non des batailles, de belles lunettes ou de beaux regards sur le monde et non des attaques contre le pouvoir et la « domination».

\section{NOTES}

1. Olivier Gosselain, «Slow science - La désexcellence », Uzance, Vol. 1, 2011, p. 131.

2. Paris, Seuil, 2006.

3. Projet Occupy \#2 - IFG : https://www.occupyartproject.com/; Viz Laboratory for Visual Culture : $\quad$ https://vizlaboratory.org/? fbclid=IwAR3BJ7JsgC0Vk-0TLcYut9pt4fc2sfI4YoP6rb2_7UZyyOhTGgYCIte09No

4. À titre indicatif : Aparamillon - https://cargocollective.com/aparamillon/About ; L'équipe de la plateforme Assosyo - https://1-2.gr/; Theatro Choros - https://www.facebook.com/ horostheatrecompany/; KET - https://polychorosket.gr/en/ ; Avtonomi Akadimia - http:// avtonomi-akadimia.net/el ; Communitism - https://communitism4art.wordpress.com/ ; Theatro Empros - https://www.embros.gr/; Enterprise Projects - http://enterpriseprojects.com/; Kassandras - http://kassandras.rehab/ Victoria Square Project, Studio http://victoriasquareproject.gr/. 
5. Voir «Lalka» de Lena Kitsopoulou, URL: https://www.onassis.org/video/lalka-lenakitsopoulou, consulté le 16 juillet 2021.

6. URL : https://thereceptionathens.eu/, consulté le 16 juillet 2021.

7. Contre le théâtre politique, Paris, La Fabrique, 2019.

\section{INDEX}

Mots-clés : projet, œuvre d'art, Grèce, statut d'artiste, capitalisme émotionel, désexcellence, post-

\section{AUTEURS}

\section{ANGELIKI POULOU}

Angeliki est une artiste-chercheuse dans le domaine des nouveaux médias et des arts du spectacle. Elle est engagée dans l'exploration de la culture numérique, en dialogue avec les nouveaux formats du performatif. Ses recherches actuelles portent sur la dramaturgie de nouveaux médias, les archives des arts numériques et les Communs. Angeliki fait partie du collectif d'art contemporain Medea Electronique (www.medeaelectronique.com). De 2017 à 2019, elle a été commissaire académique du programme européen « Drame grec antique: Approches Interdisciplinaires et inter-artistiques » à la Fondation Michael Cacoyannis à Athènes. En tant que curatrice et dramaturge, elle a collaboré avec des artistes indépendants et des institutions artistiques en France et en Grèce (Institut Français, Documenta 14, Théâtre National, Stegi Onassis, Fondation Michael Cacoyannis, Artec, Lab'bel, Mirfestival, etc.).

Diplômée du Département des Médias de l'Université Capodistrienne et Nationale d'Athènes, elle a poursuivi une maîtrise en Arts du Spectacle (Université Sorbonne Nouvelle-Paris III). Elle est titulaire d'une thèse de doctorat sur les nouveaux médias et la tragédie grecque (2017, co-tutelle : Université Sorbonne Nouvelle-Paris III \& Université Nationale et Capodistrienne d'Athènes).

\section{ESTELLE BAUDOU}

Pendant la préparation de ce numéro (2019-2021), Estelle Baudou bénéficiait d'un financement Marie Sklodowska Curie de la commission européenne pour mener sa recherche post-doctorale à l'APGRD (Archive of Performances of Greek and Roman Drama) de l'Université d'Oxford (her project has received funding from the European Union's Horizon 2020 research and innovation programme under the Marie Sklodowska-Curie grant agreement No 839770). Son travail, entre pratique et théorie, porte sur la mise en scène de la guerre et des mythes antiques en Europe depuis 1945. Elle a, auparavant, réalisé un doctorat sous la direction de Christian Biet à l'Université de Paris Nanterre (Une Archéologie du commun, Classiques Garnier, 2021). Elle enseigne aussi la dramaturgie au Conservatoire Régional de Paris et travaille à plusieurs spectacles comme dramaturge ou metteure en scène. 


\section{QUENTIN RIOUAL}

Quentin Rioual est docteur en études théâtrales de l'Université Paris Nanterre, metteur en scène et chargé de cours en Histoire de la scénographie à l'École nationale supérieure des Arts

Décoratifs de Paris. Suite à la thèse qu'il a consacrée aux premières mises en scène des pièces de Maurice Maeterlinck (1891-1919), il oriente son enseignement et sa recherche vers l'histoire sociale et matérielle du décor, depuis les réformes du tournant XIXe-XXe jusqu'à

l'écoscénographie contemporaine. Son travail comme metteur en scène s'inscrit au croisement des théâtralités du silence et des cultures queers. 